Supporting information for

\title{
Spectroelectrochemical Investigation of Double-Walled Tubular J-Aggregates of Amphiphilic Cyanine Dyes
}

Jennifer L. Lyon, ${ }^{\dagger}$ Dörthe M. Eisele ${ }^{\dagger}$ Stefan Kirstein, ${ }^{\ddagger}$ Jürgen P. Rabe, ${ }^{\ddagger}$ David A. Vanden Bout ${ }^{\dagger}$ and Keith J. Stevenson ${ }^{\dagger}$

${ }^{\dagger}$ Department of Chemistry and Biochemistry, Center for Electrochemistry, The University of

Texas at Austin, Austin, TX 78712, USA

Department of Physics, Humboldt University, Newtonstr. 15, D-12489 Berlin, Germany

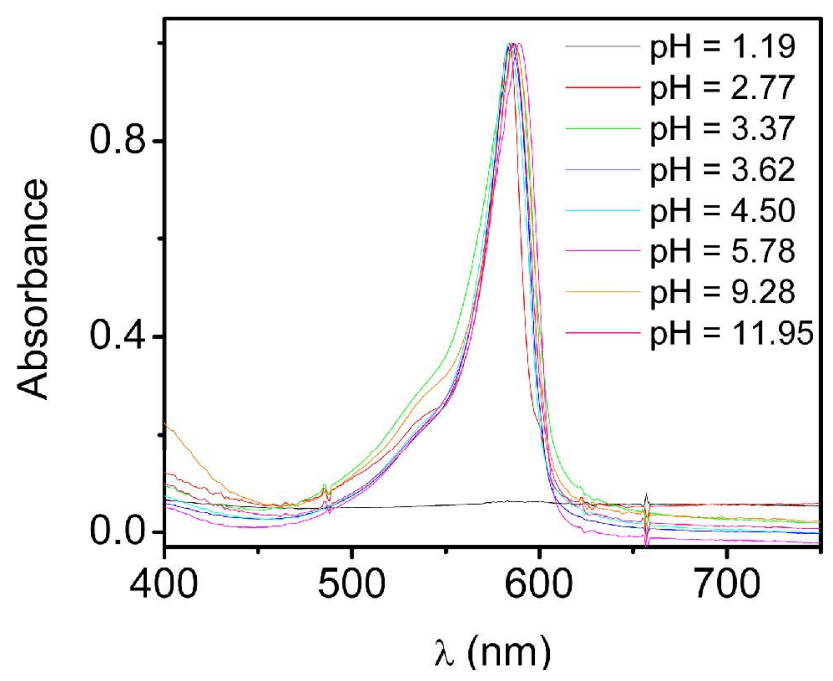

Figure S1. UV-Vis absorbance spectra of immobilized C8S3 J-aggregates on ITO immersed in $1 \mathrm{M} \mathrm{KNO}_{3}$ solutions of varying $\mathrm{pH}$, labeled above.

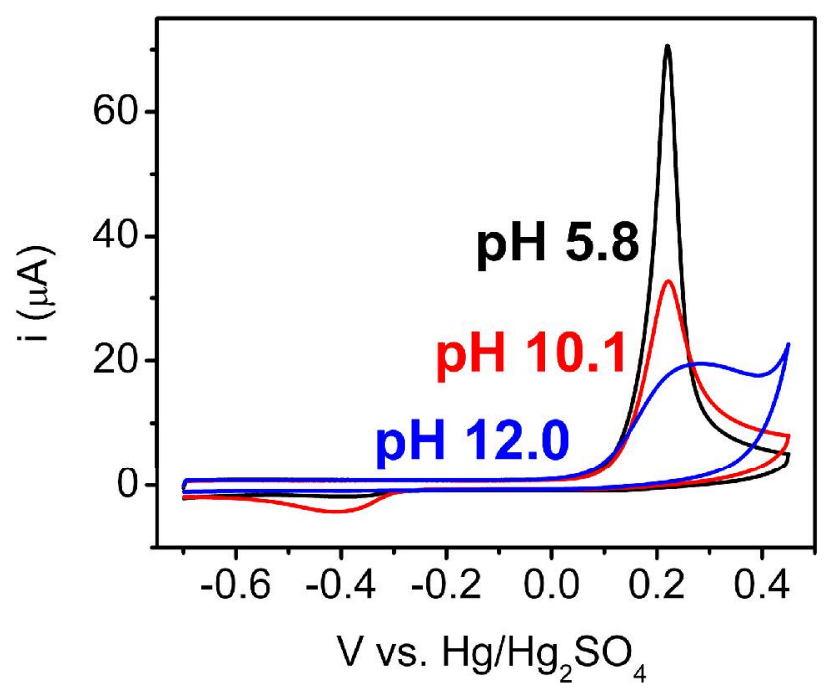

Figure S2. Representative CVs of J-aggregates at ITO immersed in neutral to basic $\mathrm{pH} 1 \mathrm{M}$ $\mathrm{KNO}_{3}$ solutions (labeled). 


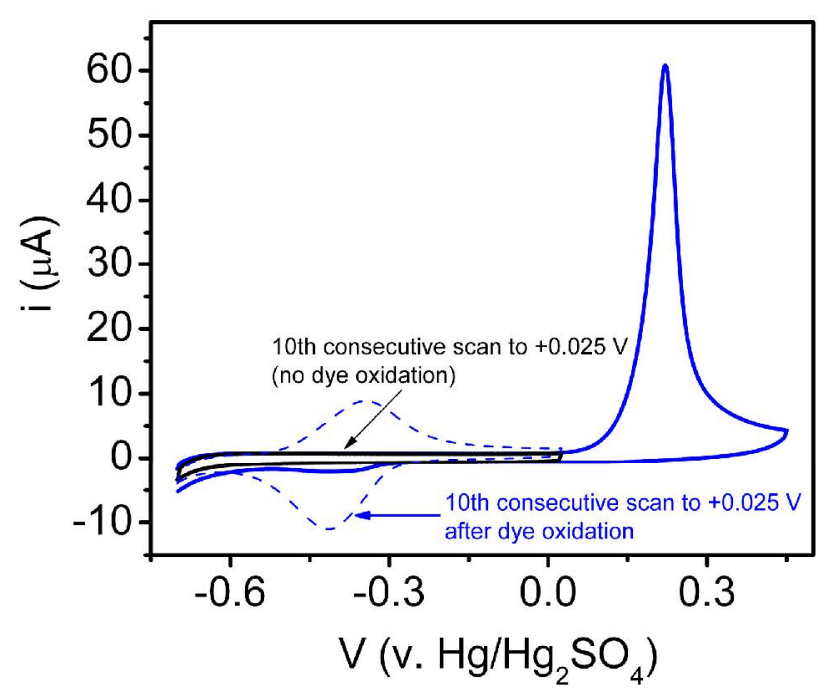

Figure S3. Comparison of voltammetric responses collected for a J-aggregate film on ITO, cycled between various potential windows for the region corresponding to the dehydrogenated dimer (DHD) redox activity, both before (solid black line) and after (dashed blue line) inducing J-aggregate oxidation by scanning the potential to $+0.45 \mathrm{~V}$ (solid blue line).

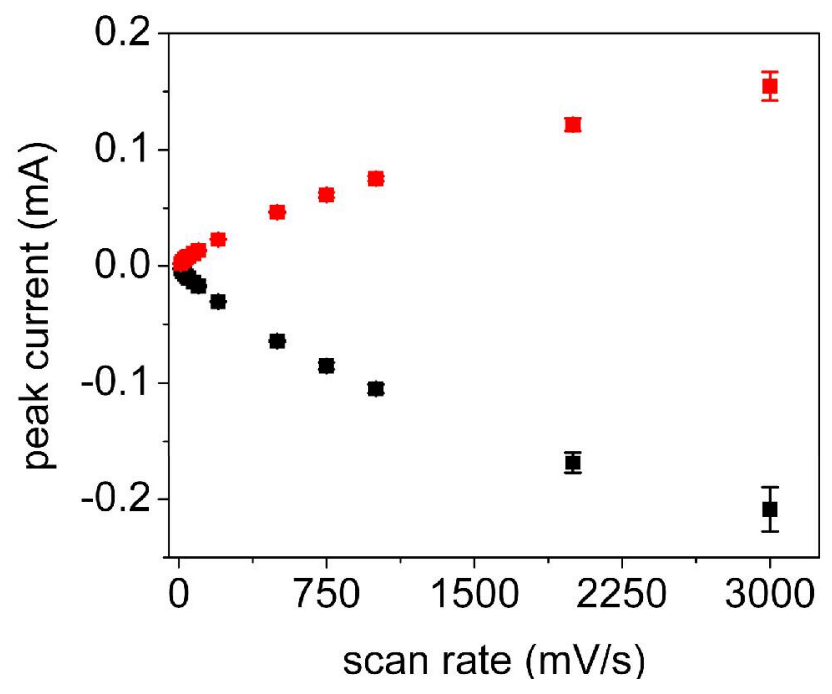

Figure S4. Peak currents (red, anodic; black; cathodic) as a function of scan rate for DHD, showing a linear dependence with scan rate. $\mathrm{N}=3$ trials at each scan rate. 


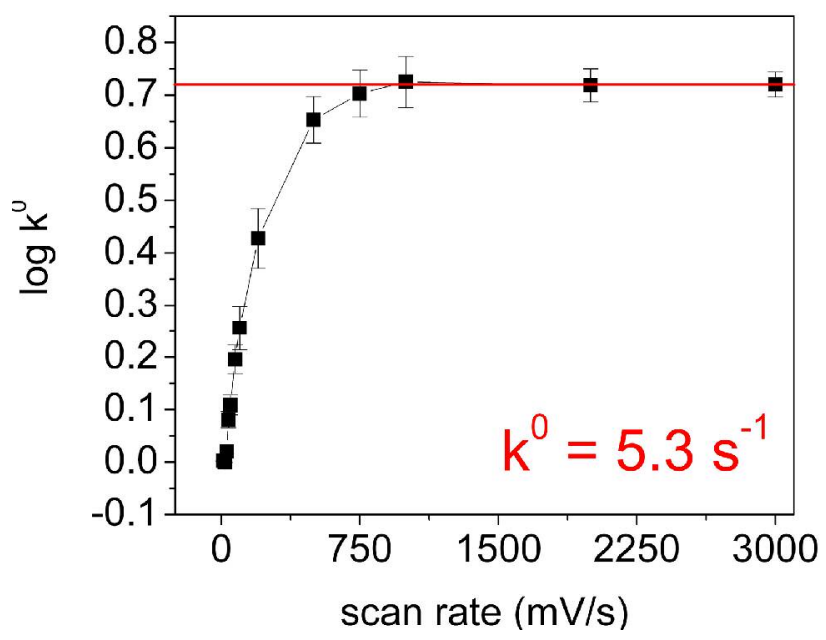

Figure S5. Plot of $\log \mathrm{k}^{0}$ versus scan rate. $\mathrm{k}^{0}{ }_{\mathrm{obs}}$ is estimated from $\Delta \mathrm{E}_{\mathrm{p}}$ data corresponding to the reversible redox response seen for DHD taken as a function of scan-rate. $\mathrm{N}=3$ trials at each scan rate.
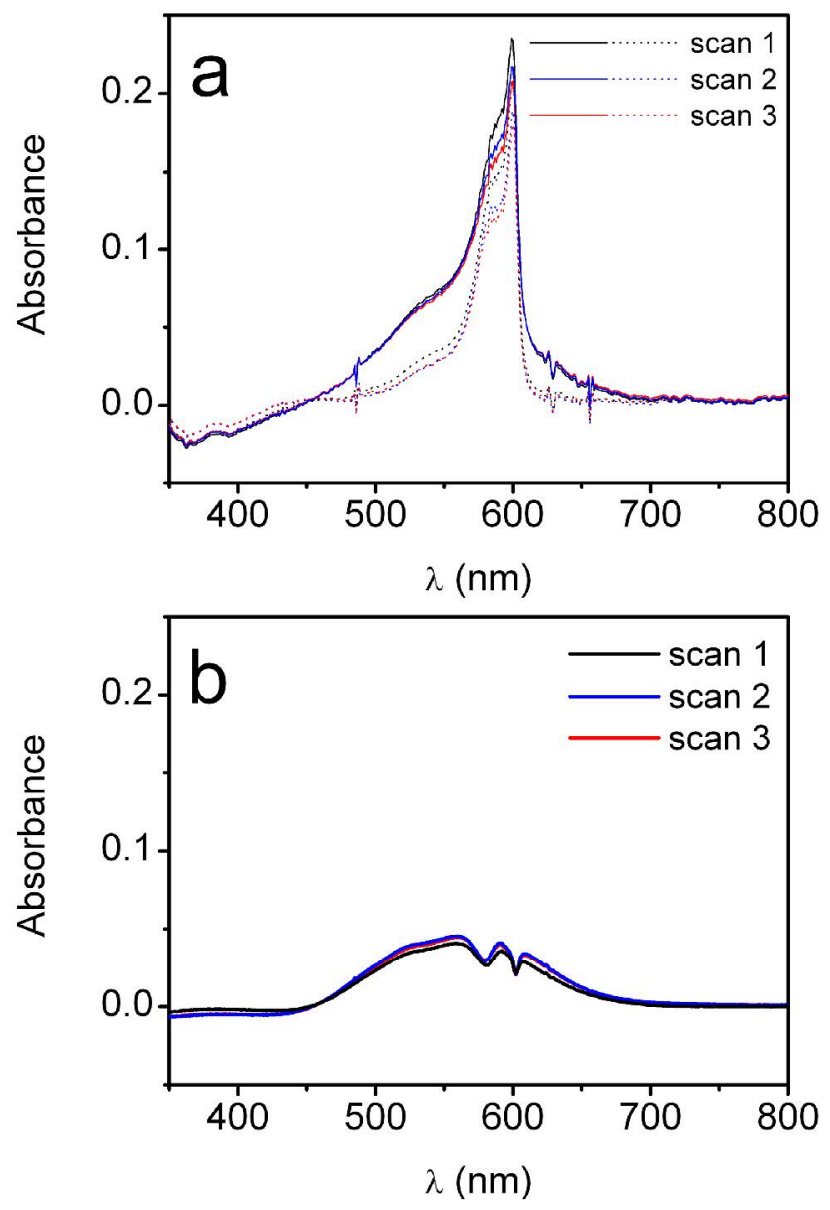

Figure S6. (a) UV-Vis spectra acquired before (solid lines) and after (dotted lines) reduction of $\mathrm{DHD}_{\mathrm{ox}}$ for each of three consecutive scans. (b) Spectra acquired by subtraction of the dotted lines from their corresponding solid lines in (a). 